\title{
PMS (Premenstrual Syndrome)
}

\section{Opinion}

Have you ever wondered if all these cramps and the lower backache, these mood-swings and the cravings is actually a normal state before your upcoming period? Thousands of women struggle to go to work or to get some sleep sometimes a whole week before their upcoming period due to an unclear syndrome which has been identified and called Premenstrual Syndrome.

If we take a closer look at the energy field after the ovulation date and when there is no fertilization we have a strong energetic impact to the brain and the fertility organs, due to the hormones that our body produces. So a clairvoyant can see or feel the forehead and ajna chakras, as well as the brain hemispheres chakras grow bigger in size but simultaneously become dirtier because of the thought forms that arise from previous experiences. The same happens to the navel, sex and basic chakras, they can be bigger and heavier, which result to dragging the apana energy down. On the other hand, prana energy is going up due to the upper chakras. The samana energy which is in the middle way goes thinner and this is when symptoms like bloating and vomiting arise. Taking into consideration that a good-balanced person tries

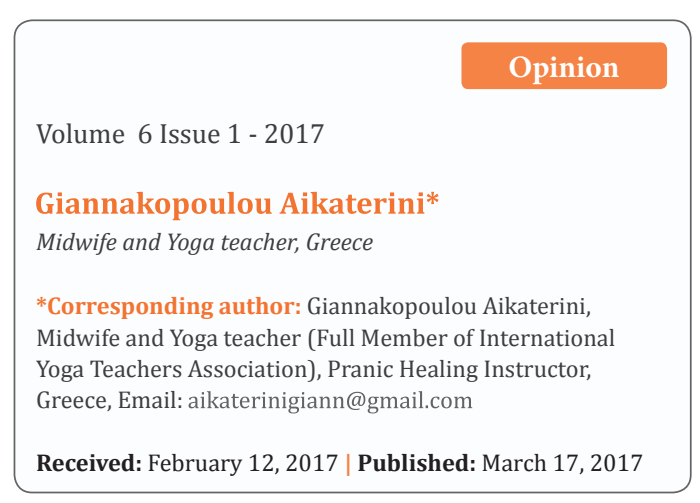

to unite prana and apana in order to stimulate samana vayu and get the balanced energy that is required for a healthy living or for meditation, one can say that the premenstrual period is working to the exact opposite way!!!

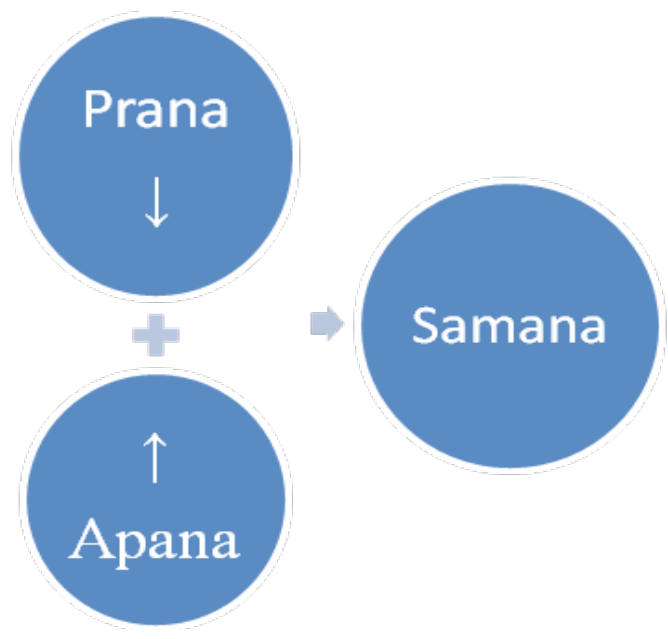

From an Ayurvedic point of view the PMS is an imbalance in the three doshas. Vata, Pitta and Kapha are severely influenced and they create a whole new, unknown constitution to the woman which results to symptoms of sickness and uncomfortness.

Vata symptoms can variate from cramps and pain to gas movement and mood changes. Pitta symptoms affect mostly the skin (pimples and redness) and create inflammatory signs like breast tenderness or feeling hot. It can also bring up feelings such as anger or depression in the form of personal blame. Finally Kapha symptoms can be water retention and weight gain, sense of heaviness or being lazy. The same person can have different imbalances or symptoms from period to period as Vikriti, as how this temporary imbalanced state is named, are very easy to affect.

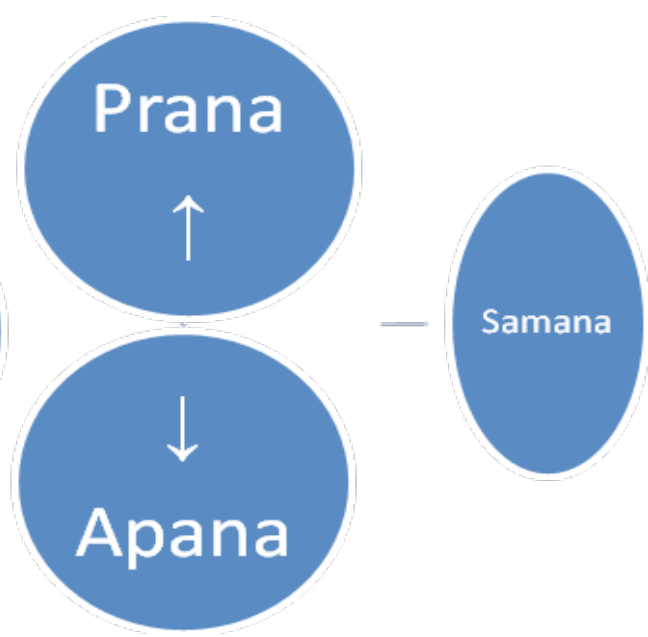

Ayurveda and yoga can be two of the major ways a woman can easily regulate and cure the PMS, only by regulating the vayus through asanas, relaxation and pranayama and the doshas through nutrition and the use of herbs. Before getting herbs, it is always useful to consult an Ayurvedic doctor or nutritionist but herbs which are involved in PMS are usually tridoshic herbs like Triphala or Shatavari but this can be quite individual and easily changeable. However nutrition is easy to regulate especially one week before the upcoming period. Try to avoid processed food, meat and dairy products, sugar and coffee or alcohol. A balanced and vegetarian diet is helpful, boiled and warm food, whole grains and fruits. Drink plenty of water and herbal teas, using herbs which alleviate your most severe symptoms. 
Asanas which turn prana vayu downwards and apana vayu upwards can help a lot. A correct exercise program would start with exercises which ground the energy like standing positions and end with inversions. It is also important to use a lot of twists so as the body can detoxify stagnant energies. A personal therapeutic program is needed, so it is important to consult a therapy yoga instructor because it will be a specific program just a week before the expected period which has to stop or change when the period comes.

Pranayama plays a very important role in all therapeutic approaches. PMS can be regulated through the Nadi Sodhana pranayama using a ratio 2:1:2:1 (Inhale-hold-exhale-hold). For those who are affected mostly psychologically, simple breathing without holds is preferable, but has to be in a ratio 1:2 (inhaleexhale). Other important breathing techniques can be Brahmari and Shitkari with long exhalations. Relaxation in Savasana and simple meditation with mantra japa can be also very helpful. It is important to minimize stress and allow all vayus to settle down and create a peaceful environment with no thoughts and no feelings.

Premenstrual syndrome is not a disease but rather a slow adjustment of the etheric and physical body to the lack of fertilization and the new menstrual cycle. Therefore taking medicins is only suppressing the physical tendency of the human body, whereas a woman must learn to listen to the body messages and to try to adapt to new habits while waiting for the body to recover and heal. There is plenty of magic and power to this feminine part of creation and a woman is called to unveil it every month! 